\title{
UMA APLICAÇÃO DA PROGRAMAÇÃO LINEAR PARA DESIGNAÇÃO DE ACADÊMICOS EM EQUUIPES DE APOIO A ORGANIZAÇÃO DE EVENTOS ACADÊMICOS: O CASO EEPA- ENPEPRO.
}

\author{
Manuel Duarte Pinheiro ${ }^{1}$ \\ Fernando do Carmo Pereira ${ }^{2}$ \\ João Luiz Rissardi ${ }^{3}$ \\ Marcos Vinicius Schwanz ${ }^{4}$ \\ Jorge Luís Nunes de Góes ${ }^{5}$
}

\begin{abstract}
RESUMO: Designar pessoal as equipes de apoio à organização de eventos acadêmicos não é uma tarefa trivial, pois é preciso considerar as preferências e as habilidades dos candidatos a comporem as equipes. O presente artigo tem como objetivo desenvolver um modelo para o problema de designação de Acadêmicos Candidatos (AC) as equipes de apoio a Comissão Organizadora do EEPA-ENPEPRO, bem como apresentar a solução ótima para o problema. Os métodos de abordagem utilizados no trabalho foram o qualitativo e quantitativo. A pesquisa para o estudo classifica-se quanto aos fins como descritiva, exploratória e explicativa, e quanto aos meios como pesquisa bibliográfica e estudo de caso. Visando identificar o número de $\mathrm{AC}$ a serem designados a cada equipe de apoio, foi desenvolvido um modelo inicial, cujos resultados serviram de base para o desenvolvimento de 3 submodelos. Com a resolução do modelo inicial e dos 3 submodelos, obteve-se o seguinte cenário de designação de $\mathrm{AC}$ às equipes de apoio: $4 \mathrm{AC}$ - Artigos; $2 \mathrm{AC}$ - Certificação; $6 \mathrm{AC}$ - Coffee Break; 1 AC - Divulgação; 2 AC - Inscrição; 1 AC - Mesa-Redonda; 3 AC - Mini-Cursos; 1 AC - Palestras; 1 AC - Patrocínio; 4 AC - Protocoloco e Cerimonial; 4 AC - Visitas Técnicas; e 1 AC - Workshop.
\end{abstract}

Palavras-chave: Pesquisa Operacional; Problemas de Designação; Programação Linear Inteira.

ABSTRACT: Designating people of support teams to academic events organization is not a trivial task because we need to consider the candidates preferences and skills to compose teams. This article aims to develop a model for the assignment problem of Candidates Academic (CA) of support teams to the Organizing Committee of EEPA-ENPEPRO and present the optimal solution to the problem. The approach methods used in the study were

\footnotetext{
${ }^{1}$ Manuel Duarte Pinheiro (Lisboa, Portugal) manuel.pinheiro@tecnico.ulisboa.pt

${ }^{2}$ Fernando do Carmo Pereira (UTFPR, Brasil) fernando_docarmo@hotmail.com

${ }^{3}$ João Luiz Rissardi (UTFPR, Brasil) luizrissardi@gmail.com

${ }^{4}$ Marcos Vinicius Schwanz (UTFPR, Brasil) mvschwanz@gmail.com

${ }^{5}$ Jorge Luís Nunes de Góes (UTFPR, Brasil) jgoes@utfpr.edu.br
}

ReLAInEP - Revista Latino-America de Inovação e Engenharia de Produção, Curitiba, PR, Brasil, v. 3, n. 4, p. 189-201, 2015. 


\section{ReLAInEP}

both qualitative and quantitative. The research for the study was classified as descriptive, exploratory and explanatory according to the purposes, and as literature and case study according to means. To identify the number of CA to be assigned to each support team, an initial model, whose results were basis for the development of three sub-models, was developed. With the resolution of the initial model and the three sub-models, we obtained the following scene of the CA support teams designation: CA 4 - Articles; CA 2 - Certification; 6 CA - Coffee Break; CA 1 - Propagation; CA 2 - Registration; CA 1 - Roundtable; 3 CA Mini-Courses; CA 1 - Lectures; 1 CA - Sponsorship; 4 CA - Protocol and Ceremonial; 4 CA Technical Visits; and $1 \mathrm{CA}$ - Workshop.

Keywords: Operational Research; Problems of Designation; Integer Linear Programming.

RESUMEN: Designar pessoal as equipes de apoio à organização de eventos acadêmicos não é uma tarefa trivial, pois é preciso considerar as preferências e as habilidades dos candidatos a comporem as equipes. $\mathrm{O}$ presente artigo tem como objetivo desenvolver um modelo para o problema de designação de Acadêmicos Candidatos (AC) as equipes de apoio a Comissão Organizadora do EEPA-ENPEPRO, bem como apresentar a solução ótima para o problema. Os métodos de abordagem utilizados no trabalho foram o qualitativo e quantitativo. A pesquisa para o estudo classifica-se quanto aos fins como descritiva, exploratória e explicativa, e quanto aos meios como pesquisa bibliográfica e estudo de caso. Visando identificar o número de $\mathrm{AC}$ a serem designados a cada equipe de apoio, foi desenvolvido um modelo inicial, cujos resultados serviram de base para o desenvolvimento de 3 submodelos. Com a resolução do modelo inicial e dos 3 submodelos, obteve-se o seguinte cenário de designação de $\mathrm{AC}$ às equipes de apoio: $4 \mathrm{AC}$ - Artigos; $2 \mathrm{AC}$ - Certificação; $6 \mathrm{AC}$ - Coffee Break; 1 AC - Divulgação; 2 AC - Inscrição; 1 AC - Mesa-Redonda; 3 AC - Mini-Cursos; 1 AC - Palestras; 1 AC - Patrocínio; 4 AC - Protocoloco e Cerimonial; 4 AC - Visitas Técnicas; e 1 AC - Workshop.

Palabras-clave: Pesquisa Operacional; Problemas de Designação; Programação Linear Inteira.

\section{INTRODUÇÃO}

A Programação Linear foi desenvolvida conceitualmente após a Segunda Guerra Mundial, pelo soviético Kolmogorov, com o objetivo de resolver problemas militares de logística. A primeira aplicação da Programação Linear foi feita em 1945, por Stigler em um problema referente à composição de uma mistura (MORAIS, 2013).

Em modelos de Programação Linear todas as relações matemáticas devem ser lineares, ou seja, um modelo de programação linear é um modelo de matemático de otimização no qual todas as funções são lineares, conforme afirma Goldbarg e Luna (2005).

De acordo com Arenales et al. (2007) exemplos de problemas que podem ser formulados como um problema de Programação Linear aparecem nas mais variadas áreas, dentre as quais, destacam-se: problemas de mistura, problemas de transporte, problemas de transbordo, problemas de designação/atribuição, problemas de planejamento da produção, problemas de programação de projetos, problemas de fluxo de caixa, problemas de meio ambiente, problemas de corte e empacotamento, entre outros.

ReLAInEP - Revista Latino-America de Inovação e Engenharia de Produção, Curitiba, PR, Brasil, v. 3, n. 4, p. 189-201, 2015. 


\section{ReLAInEP}

Dentre as várias categorias de problemas de Programação Linear, conforme anteriormente citado, estão os problemas de designação ou atribuição. A designação de tarefas a pessoas consiste no processo em que se definem quais recursos humanos serão utilizados para executar as tarefas que compõem um determinado projeto, respondendo basicamente a questão: "Quem deve fazer isso?" Considerando critérios de alocação para selecionar os mais adequados, baseados nos objetivos de negócio da organização (MARQUES et al., 2011).

Os modelos de designação ou atribuição são modelos de Programação Inteira, um caso particular dos modelos de Programação Linear. Tem-se um problema de Programação Linear Inteira (PLI), quando todas as variáveis do modelo para a resolução do problema não assumem valores contínuos e sendo possível somente a utilização de valores discretos (GOLDBARG; LUNA, 2005).

\section{PROGRAMAÇÃO LINEAR E PROBLEMAS DE DESIGNAÇÃO}

Um problema de programação linear inteira pode apresentar as seguintes situações (SUCENA, 2012): i) Todas as variáveis de decisões são inteiras (Problemas de Programação Linear Inteira Pura - PLIP); ii) Parte das variáveis de decisões são inteiras (Problemas de Programação Linear Inteira Mista - PLIM); iii) Todas as variáveis de decisões são binárias (Problemas de Programação Linear Inteira Binária - PLIB); e iv) Parte das variáveis de decisões são binárias (Problemas de Programação Linear Inteira Binária Mista - PLIBM).

Os modelos de designação de tarefas a pessoas são modelos em que as variáveis Xij podem assumir apenas valores 0 ou 1 . Um ponto do domínio $\mathrm{Z}$ (função objetivo), sujeita às restrições citadas anteriormente, corresponde a uma alocação de tarefas, sendo a imagem de $\mathrm{Z}$ naquele ponto, o custo de alocação. Quando uma alocação é efetuada em relação à minimização ou maximização de Z, se tem uma alocação ótima de tarefas (RODRIGUES; VIEIRA; AGUSTINI, 2005).

Supondo um problema com $n$ tarefas que necessitam ser atribuídas a n pessoas em que pij mede o interesse do indivíduo i na realização da tarefa j e Xij representa a variável de decisão igual a 1 se o individuo $\mathrm{i}$ for designado para a realização da tarefa $\mathrm{j}$ e 0 , caso contrário. A formulação do problema do problema de designação ou atribuição, segundo Arenales et al. (2007) pode ser representada como:

$$
\begin{aligned}
& \text { Maximizar } f\left(X_{11}, \ldots, X_{n n}\right)=\sum_{i=1}^{n} \sum_{j=1}^{n} p_{i j} X_{i j} \\
& \sum_{i=1}^{n} X i j=1, i=1,2, \ldots, n \\
& \sum_{i=1}^{n} X i j=1, j=1,2, \ldots, n \\
& X i j=0 \text { ou } 1, i=1, \ldots . n, j=1, \ldots n
\end{aligned}
$$

A função-objetivo representada em (1) representa o interesse total a ser maximizado, O conjunto de restrições (2) impõem que cada indivíduo é designado a uma e somente uma tarefa. O conjunto de restrições (3) impõem que cada tarefa é realizada por apenas um e somente um indivíduo. As restrições (4) impõem que as variáveis de decisão tomem os

ReLAInEP - Revista Latino-America de Inovação e Engenharia de Produção, Curitiba, PR, Brasil, v. 3, n. 4, p. 189-201, 2015. 


\section{ReLAInEP}

valores 0 ou 1 (ARENALES et al., 2007). No entanto, Arenales et al. (2007) afirmam que devidos a características particulares da estrutura de um modelo de designação, as restrições (2) podem ser substituídas por $\mathrm{Xij} \geq 0$ e, mesmo assim, pode-se mostrar que o modelo tem solução ótima inteira.

Marques et al. (2011) destacam que identificar a atribuição mais adequada não é uma solução trivial, desta forma, são necessários mecanismos que permitam analisar as diversas possibilidades de alocação com critérios claramente definidos.

Modelos típicos de programação linear na prática podem envolver muitas variáveis e restrições, sendo, portanto, o uso de computadores o único modelo de resolver tais modelos (TAHA, 2008). Existe uma série de softwares específicos para a resolução de problemas de programação linear, sendo o LINDO um dos mais populares, segundo Lanchtermacher (2007). O Solver do Excel é particularmente atraente para usuário de planilhas (TAHA, 2008). No entanto, o Solver do Excel apresenta limitações em relação ao número de variáveis do problema, porém para os propósitos deste estudo, o Solver do Excel mostrou-se adequado.

De acordo com Lanchtermacher (2007), para modelar um problema de programação linear na planilha do Excel, devemos primeiramente designar células para representar cada uma das entidades a seguir: i) função-objetivo; ii) variáveis de decisão; iii) restrições; iv) equações que representam cada uma das restrições do problema (LHS - Left Hand Side); e v) valor associado a cada restrição (RHS - Right Hand Side), com por exemplo, disponibilidade de um determinado recurso. A Figura 1 ilustra a modelagem do problema na planilha do Excel, bem como as fórmulas utilizadas na modelagem do problema.

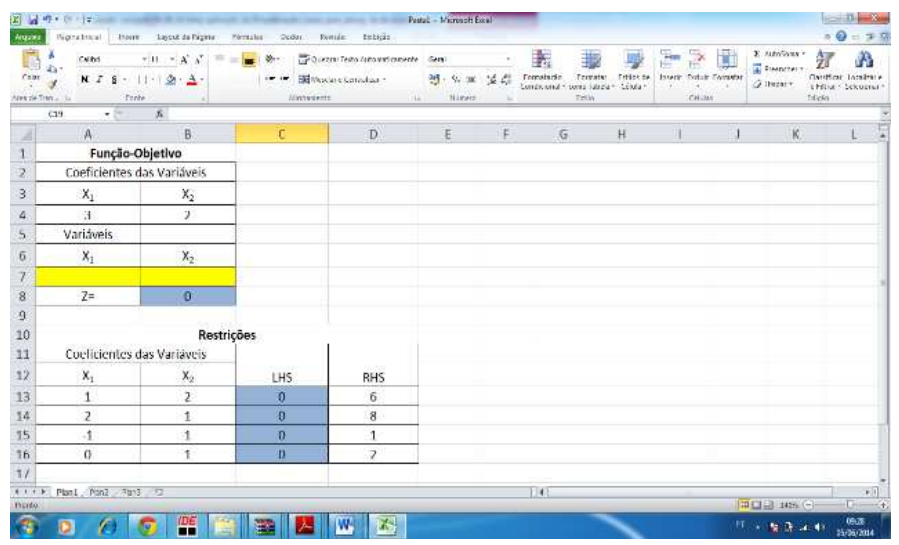

FIGURA 1 - Modelagem do problema no Excel. Fonte: Adaptado de Lanchtermacher (2008)

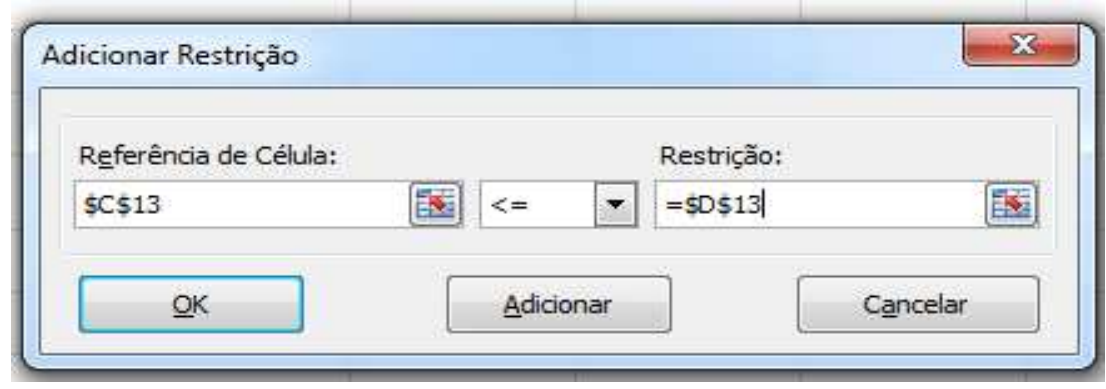

FIGURA 2 - Apresenta a tela dos parâmetros do Solver do Excel.

ReLAInEP - Revista Latino-America de Inovação e Engenharia de Produção, Curitiba, PR, Brasil, v. 3, n. 4, p. 189-201, 2015. 


\section{ReLAInEP}

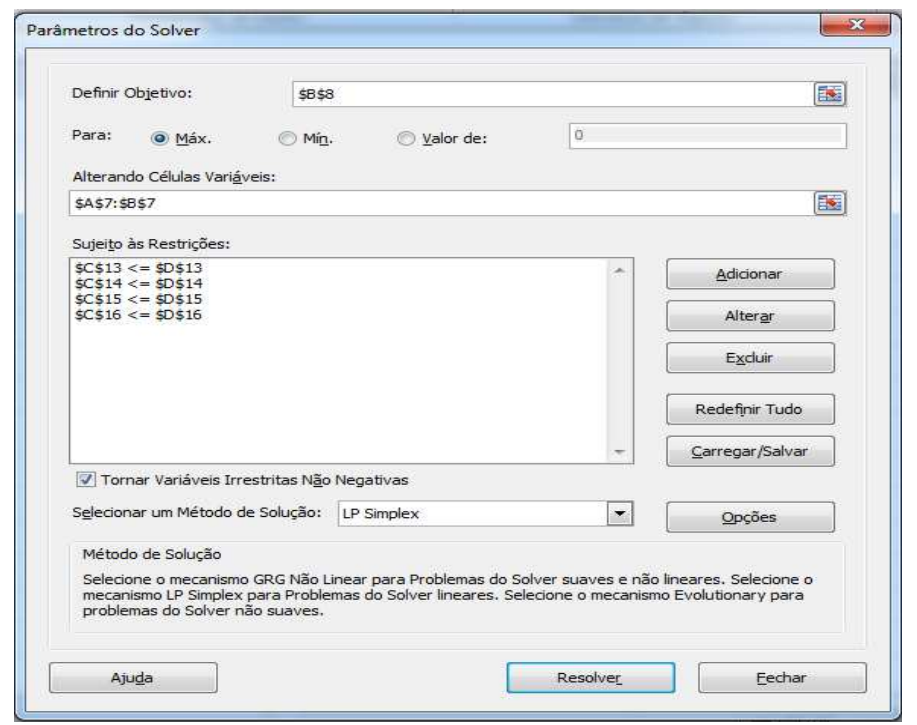

FIGURA 3 - Designando restrições no Solver do Excel. Fonte: Solver do Excel.

Após designadas todas as restrições do problema, devemos garantir que todas as variáveis sejam não negativas, selecionando a opção "tornar variáveis irrestritas não negativas". E por fim, no Campo "Selecionar um Método de Solução", devemos selecionar para o caso de problemas de programação linear o Método LP Simplex.

\section{REVISÃO DE LITERATURA}

A revisão de literatura teve como foco a busca por artigos que tratassem de estudos realizados sobre alocação de equipes através da aplicação da Programação Linear. A busca foi feita nos portais Capes, Scielo e buscador Google.

Barbosa (2009) propõe um modelo híbrido estruturado em programação matemática multicritério, com o objetivo de apoiar a organização de Tecnologia da Informação. O modelo foi aplicado em uma empresa que desenvolve projetos de pesquisa e desenvolvimento em tecnologia da informação, visando ao aumento de produtividade com a maximização da alocação dos recursos, a minimização das folgas e a redução de custos do produto final. $\mathrm{O}$ método utilizado foi o Measuring Attractiveness by a Categorical Based Evaluation Technique (MACBETH).

Costa (2009) demonstra como a utilização de um modelo matemático pode contribuir para auxiliar os gerentes de projeto na fase de alocação de recursos humanos, especificamente no contexto de projetos de Sistemas de Informação. O modelo utilizado no estudo é caracterizado por 5 restrições: i) um profissional não pode executar outros projetos paralelamente; ii) cada profissional possui habilidades diferentes para execução de cada projeto; iii) as habilidades são definidas em níveis de 0 (zero) a 1 (um); e iv) a alocação do profissional é dada em função do tempo de execução da tarefa bem como o custo para execução da mesma tarefa.

Teixeira (2011) desenvolveu um modelo capaz de determinar a melhor distribuição de vagões entre as cargas de clientes que demandam atendimento, assim otimizando os recursos

ReLAInEP - Revista Latino-America de Inovação e Engenharia de Produção, Curitiba, PR, Brasil, v. 3, n. 4, p. 189-201, 2015. 


\section{ReLAInEP}

disponíveis em cada situação, gerando a maior margem por vagão hora possível. O modelo vai contemplar as restrições: número de vagões gôndolas disponíveis, os volumes de cargas para o transporte, as capacidades dos terminais de carga e de descarga, os períodos de retorno dos vagões para carregamento e os dias disponíveis para o transporte.

Silva et al. (2014) desenvolveu um modelo matemático computacional destinado a fazer a alocação de artigos em sessões de eventos para ser apresentado. O modelo desenvolvido é caracterizado pelas seguintes restrições: i) cada sessão deve receber no mínimo três avaliadores de artigos; ii) cada sessão deve receber no máximo sete apresentações de artigos; iii) cada sessão deve receber artigos pertencentes às mesmas temáticas; e iv) cada sessão deve ser alocada a uma única sala.

\section{METODOLOGIA}

Os métodos de abordagem utilizados foram o qualitativo e quantitativo. A pesquisa para o estudo classifica-se quanto aos fins como descritiva, exploratória e explicativa, e quanto aos meios como pesquisa bibliográfica e estudo de caso.

Foi elaborada uma planilha com o nome dos acadêmicos que se candidataram para compor as equipes de apoio à comissão organizadora, sendo necessário que cada candidato atribuísse notas de 0 a 10 para cada equipe de acordo com sua afinidade. Com esses dados foi desenvolvido um modelo de programação linear, e o mesmo foi resolvido no Solver do Excel, disponível no pacote Office 2010. Foi utilizado um computador Acer®, com processador Intel Core i5-2450M com $2.5 \mathrm{GHz}$ e 4 GB de memória RAM.

Para a resolução do problema utilizando o Solver do Excel, inicialmente foram agrupadas algumas equipes de apoio, pois o Solver do Excel só comporta 200 variáveis de decisão. Inicialmente foi elaborado um modelo inicial que agregou algumas equipes. Com base nos resultados do modelo inicial, foram criados submodelos para designar os acadêmicos as equipes individuais, ou seja, foram desagrupadas as equipes de apoio que no modelo inicial estavam agrupadas.

\section{ESTUDO DE CASO}

O VIII Encontro de Engenharia de Produção Agroindustrial da UNESPAR/Campus de Campo Mourão (VIII EEPA) e o II Encontro Paranaense de Engenharia de Produção (II ENPEPRO) têm por objetivo proporcionar um espaço para discutir, consolidar e expandir conhecimentos da área de Engenharia de Produção no cenário regional e estadual.

O VIII EEPA constitui um evento promovido pelo Curso de Engenharia de Produção Agroindustrial da UNESPAR/Campus de Campo Mourão e orientado à integração entre profissionais, discentes, docentes, pesquisadores das áreas de Engenharia de Produção, Alimentos, Agropecuária e áreas afins e tem por finalidade levar aos acadêmicos, conhecimentos complementares à grade curricular do Curso. O II ENPEPRO é um evento de abrangência estadual, idealizado pelo Núcleo Paranaense de Estudantes de Engenharia de Produção e destinado ao aprimoramento dos conhecimentos técnicos que visam integrar acadêmicos, pesquisadores, profissionais liberais e a comunidade acadêmica para uma avaliação e prospecção de oportunidades e desafios aos profissionais da área de Engenharia de Produção. Uma vez que o VIII EEPA e o II ENPREPRO serão organizados simultaneamente

ReLAInEP - Revista Latino-America de Inovação e Engenharia de Produção, Curitiba, PR, Brasil, v. 3, n. 4, p. 189-201, 2015. 


\section{ReLAInEP}

e realizados nos dias 16 e 17 de Setembro de 2014, doravante, os eventos serão referenciados neste estudo, como Evento.

A comissão organizadora do Evento é composta por 12 equipes de apoio. A relação de equipes e descrição das respectivas atividades são apresentadas no Quadro 1 a seguir.

QUADRO 1 - Descrição das equipes dos eventos.

\begin{tabular}{|c|c|}
\hline Equipe & Descrição das Atividades \\
\hline Artigos (AT) & $\begin{array}{l}\text { Receber e encaminhar artigos para avaliadores e preparar anais do } \\
\text { Evento. }\end{array}$ \\
\hline Certificação (CT) & Preparar toda a certificação do Evento. \\
\hline Coffe Break (CB) & Organizar os coffe breaks do Evento. \\
\hline Divulgação (DV) & Realizar a divulgação do Evento. \\
\hline Inscrição (IN) & Receber e processar as inscrições de artigos e de ouvintes do Evento \\
\hline Mesa-Redonda (MR) & $\begin{array}{l}\text { Definir Mesa-Redonda e organizar procedimentos para } \\
\text { operacionalização. }\end{array}$ \\
\hline Mini-Cursos (MC) & $\begin{array}{l}\text { Definir Mini-Cursos e organizar procedimentos para } \\
\text { operacionalização. }\end{array}$ \\
\hline Palestras (PL) & Definir Palestras e organizar procedimentos para operacionalização. \\
\hline Patrocínio (PT) & Angariar patrocínios para o Evento. \\
\hline $\begin{array}{l}\text { Protocolo e Cerimonial } \\
\text { (PC) }\end{array}$ & Elaborar e executar o cerimonial do Evento. \\
\hline Visitas Técnicas (VT) & $\begin{array}{l}\text { Definir Visitas Técnicas e organizar procedimentos para } \\
\text { operacionalização. }\end{array}$ \\
\hline Workshop (WS) & $\begin{array}{l}\text { Definir Workshops e organizar procedimentos para } \\
\text { operacionalização. }\end{array}$ \\
\hline
\end{tabular}

Fonte: Elaborado pelos autores.

Para a resolução do problema de alocação de Acadêmicos Candidatos (AC) as equipes de apoio a Comissão Organizadora do Evento, levando em consideração a preferência do acadêmico a uma equipe em detrimento de outra, foi necessário gerar uma matriz de pesos. 28 acadêmicos que se candidataram para compor as equipes, sendo que cada equipe necessita de pelo menos 1 candidato. Devido às limitações do Solver do Excel, em termos de números de variáveis de decisão, inicialmente algumas das 12 equipes de apoio foram agrupadas, conforme apresentado na Tabela 1 .

ReLAInEP - Revista Latino-America de Inovação e Engenharia de Produção, Curitiba, PR, Brasil, v. 3, n. 4, p. 189-201, 2015. 


\section{ReLAInEP}

TABELA 1 - Matriz de pesos conforme preferência dos acadêmicos.

\begin{tabular}{|c|c|c|c|c|c|c|c|}
\hline Acadêmicos/Equipes & PT/DV & $\mathrm{IN} / \mathrm{CT}$ & $\begin{array}{c}\mathrm{PT} / \mathrm{WS} / \mathrm{M} \\
\mathrm{R}\end{array}$ & $\begin{array}{c}\mathrm{VT} / \mathrm{M} \\
\mathrm{C}\end{array}$ & $\mathrm{PC}$ & $\mathrm{CB}$ & $\mathrm{AT}$ \\
\hline $\mathrm{AC} 1$ & 6 & 7 & 7 & 7 & 6 & 8 & 7 \\
\hline $\mathrm{AC} 2$ & 5 & 10 & 6 & 6 & 8 & 10 & 7 \\
\hline AC3 & 8 & 2 & 7 & 7 & 7 & 6 & 3 \\
\hline $\mathrm{AC} 4$ & 9 & 9 & 8 & 10 & 6 & 8 & 7 \\
\hline AC5 & 3 & 4 & 7 & 5 & 10 & 5 & 6 \\
\hline AC6 & 5 & 10 & 9 & 10 & 1 & 10 & 7 \\
\hline AC7 & 5 & 10 & 4 & 4 & 4 & 10 & 8 \\
\hline AC8 & 6 & 7 & 8 & 7 & 10 & 4 & 5 \\
\hline $\mathrm{AC} 9$ & 7 & 4 & 7 & 7 & 9 & 4 & 6 \\
\hline AC10 & 2 & 3 & 6 & 9 & 8 & 2 & 8 \\
\hline $\mathrm{AC} 11$ & 6 & 8 & 7 & 6 & 6 & 7 & 6 \\
\hline $\mathrm{AC} 12$ & 4 & 8 & 5 & 6 & 7 & 7 & 9 \\
\hline $\mathrm{AC} 13$ & 1 & 5 & 3 & 4 & 6 & 10 & 6 \\
\hline $\mathrm{AC} 14$ & 3 & 4 & 7 & 8 & 5 & 9 & 10 \\
\hline AC15 & 6 & 8 & 6 & 9 & 6 & 8 & 5 \\
\hline AC16 & 8 & 6 & 7 & 8 & 8 & 5 & 6 \\
\hline $\mathrm{AC} 17$ & 4 & 8 & 4 & 7 & 5 & 3 & 5 \\
\hline AC18 & 8 & 7 & 8 & 8 & 8 & 9 & 7 \\
\hline AC19 & 8 & 7 & 6 & 8 & 4 & 8 & 6 \\
\hline AC20 & 9 & 3 & 6 & 8 & 9 & 1 & 8 \\
\hline $\mathrm{AC} 21$ & 5 & 7 & 6 & 5 & 8 & 3 & 10 \\
\hline $\mathrm{AC} 22$ & 8 & 7 & 7 & 6 & 6 & 4 & 8 \\
\hline $\mathrm{AC} 23$ & 5 & 9 & 8 & 8 & 4 & 10 & 3 \\
\hline $\mathrm{AC} 24$ & 7 & 8 & 5 & 6 & 4 & 7 & 7 \\
\hline AC25 & 6 & 6 & 6 & 9 & 3 & 6 & 5 \\
\hline AC26 & 8 & 8 & 10 & 10 & 7 & 8 & 7 \\
\hline AC27 & 7 & 5 & 1 & 3 & 3 & 7 & 1 \\
\hline AC28 & 5 & 8 & 9 & 10 & 8 & 5 & 5 \\
\hline
\end{tabular}

Fonte: Elaborado pelos autores.

$\mathrm{Na}$ formulação matemática do problema investigado, a função-objetivo (5) visa maximizar a satisfação a satisfação dos candidatos na participação das equipes de apoio. $\mathrm{Na}$ formulação matemática foram considerados os seguintes conjuntos de restrições, conforme segue: (6) indicam que em cada equipe de apoio deve ser alocado pelo menos um candidato; (7) indicam que cada candidato só pode fazer parte de uma equipe de apoio; (8) indicam o domínio das variáveis de decisão. A formulação matemática que representa o problema investigado é a seguir apresentada.

$\operatorname{Maximizar} \mathrm{Z}=\sum_{i=1}^{n} \sum_{j=1}^{m} p_{i j} X_{i j}$

ReLAInEP - Revista Latino-America de Inovação e Engenharia de Produção, Curitiba, PR, Brasil, v. 3, n. 4, p. 189-201, 2015. 


\section{ReLAInEP}

$\sum_{i=1}^{n} X i j \geq 1, \forall j=1, \ldots, m$

$\sum_{i=1}^{m} X i j=1, \forall i=1, \ldots, n$

$X i j \in\{0,1\}, \forall i=1, \ldots n, \forall j=1, \ldots m$

Em que: $n$ é número de candidato; $m$ é número de equipes de apoio; pij - peso atribuído pelo candidato i para a equipe j; e Xij = variáveis de decisão que assumem 1 , se o candidato i for atribuído a equipe j e 0 , caso contrário.

Com base nos dados da Tabela 1 o problema foi modelado no Excel, e posteriormente resolvido utilizando a ferramenta Solver. A Figura 4 ilustra a modelagem do problema na planilha do Excel.

FIGURA 4- Modelagem do problema investigado no Excel.

\begin{tabular}{|c|c|c|c|c|c|c|c|}
\cline { 2 - 8 } \multicolumn{1}{c|}{} & \multicolumn{7}{|c|}{ Dados de Entrada } \\
\hline $\mathbf{A C}_{\mathbf{E} Q}$ & $\mathbf{P T}$ & $\mathbf{I N}$ & $\mathbf{P L}$ & $\mathbf{V T}$ & $\mathbf{P C}$ & $\mathbf{C B}$ & $\mathbf{A T}$ \\
\hline $\mathbf{A C}_{\mathbf{1}}$ & 6 & 7 & 7 & 7 & 6 & 8 & 7 \\
\hline $\mathbf{A C}_{\mathbf{2}}$ & 5 & 10 & 6 & 6 & 8 & 10 & 7 \\
\hline $\mathbf{A C}_{\mathbf{3}}$ & 8 & 2 & 7 & 7 & 7 & 6 & 3 \\
\hline $\mathbf{A C}_{\mathbf{4}}$ & 9 & 9 & 8 & 10 & 6 & 8 & 7 \\
\hline $\mathbf{A C}_{\mathbf{5}}$ & 3 & 4 & 7 & 5 & 10 & 5 & 6 \\
\hline $\mathbf{A C}_{\mathbf{6}}$ & 5 & 10 & 9 & 10 & 1 & 10 & 7 \\
\hline $\mathbf{A C}_{\mathbf{7}}$ & 5 & 10 & 4 & 4 & 4 & 10 & 8 \\
\hline $\mathbf{A C}_{\mathbf{8}}$ & 6 & 7 & 8 & 7 & 10 & 4 & 5 \\
\hline $\mathbf{A C}_{\mathbf{9}}$ & 7 & 4 & 7 & 7 & 9 & 4 & 6 \\
\hline $\mathbf{A C}_{\mathbf{1 0}}$ & 2 & 3 & 6 & 9 & 8 & 2 & 8 \\
\hline $\mathbf{A C}_{\mathbf{1 1}}$ & 6 & 8 & 7 & 6 & 6 & 7 & 6 \\
\hline $\mathbf{A C}_{\mathbf{1 2}}$ & 4 & 8 & 5 & 6 & 7 & 7 & 9 \\
\hline $\mathbf{A C}_{\mathbf{1 3}}$ & 1 & 5 & 3 & 4 & 6 & 10 & 6 \\
\hline $\mathbf{A C}_{\mathbf{1 4}}$ & 3 & 4 & 7 & 8 & 5 & 9 & 10 \\
\hline $\mathbf{A C}_{\mathbf{1 5}}$ & 6 & 8 & 6 & 9 & 6 & 8 & 5 \\
\hline $\mathbf{A C}_{\mathbf{1 6}}$ & 8 & 6 & 7 & 8 & 8 & 5 & 6 \\
\hline $\mathbf{A C}_{\mathbf{1 7}}$ & 4 & 8 & 4 & 7 & 5 & 3 & 5 \\
\hline $\mathbf{A C}_{\mathbf{1 8}}$ & 8 & 7 & 8 & 8 & 8 & 9 & 7 \\
\hline $\mathbf{A C}_{\mathbf{1 9}}$ & 8 & 7 & 6 & 8 & 4 & 8 & 6 \\
\hline $\mathbf{A C}_{\mathbf{2 0}}$ & 9 & 3 & 6 & 8 & 9 & 1 & 8 \\
\hline $\mathbf{A C}_{\mathbf{2 1}}$ & 5 & 7 & 6 & 5 & 8 & 3 & 10 \\
\hline $\mathbf{A C}_{\mathbf{2 2}}$ & 8 & 7 & 7 & 6 & 6 & 4 & 8 \\
\hline $\mathbf{A C}_{\mathbf{2 3}}$ & 5 & 9 & 8 & 8 & 4 & 10 & 3 \\
\hline $\mathbf{A C}_{\mathbf{2 4}}$ & 7 & 8 & 5 & 6 & 4 & 7 & 7 \\
\hline $\mathbf{A C}_{\mathbf{2 5}}$ & 6 & 6 & 6 & 9 & 3 & 6 & 5 \\
\hline $\mathbf{A C}_{\mathbf{2 6}}$ & 8 & 8 & 10 & 10 & 7 & 8 & 7 \\
\hline $\mathbf{A C}_{\mathbf{2 7}}$ & 7 & 5 & 1 & 3 & 3 & 7 & 1 \\
\hline $\mathbf{A C}_{\mathbf{2 8}}$ & 5 & 8 & 9 & 10 & 8 & 5 & 5 \\
\hline & & & & & \\
\hline
\end{tabular}

$\operatorname{MAX} \mathrm{Z}=0$

\begin{tabular}{|c|c|c|c|c|c|c|c|c|c|}
\hline & \multicolumn{7}{|c|}{ Alocações } & \multirow[b]{2}{*}{ LHS } & \multirow[b]{2}{*}{ Disp. } \\
\hline AC/EQ & PT & IN & PL & VT & PC & CB & AT & & \\
\hline $\mathrm{AC}_{1}$ & & & & & & & & 0 & 1 \\
\hline $\mathrm{AC}_{2}$ & & & & & & & & 0 & 1 \\
\hline $\mathrm{AC}_{3}$ & & & & & & & & 0 & 1 \\
\hline $\mathrm{AC}_{4}$ & & & & & & & & 0 & 1 \\
\hline $\mathrm{AC}_{5}$ & & & & & & & & 0 & 1 \\
\hline $\mathrm{AC}_{6}$ & & & & & & & & 0 & 1 \\
\hline $\mathbf{A C}_{7}$ & & & & & & & & 0 & 1 \\
\hline $\mathrm{AC}_{8}$ & & & & & & & & 0 & 1 \\
\hline $\mathrm{AC}_{9}$ & & & & & & & & 0 & 1 \\
\hline $\mathrm{AC}_{10}$ & & & & & & & & 0 & 1 \\
\hline $\mathrm{AC}_{11}$ & & & & & & & & 0 & 1 \\
\hline $\mathrm{AC}_{12}$ & & & & & & & & 0 & 1 \\
\hline $\mathrm{AC}_{13}$ & & & & & & & & 0 & 1 \\
\hline $\mathrm{AC}_{14}$ & & & & & & & & 0 & 1 \\
\hline $\mathrm{AC}_{15}$ & & & & & & & & 0 & 1 \\
\hline $\mathrm{AC}_{16}$ & & & & & & & & 0 & 1 \\
\hline $\mathrm{AC}_{17}$ & & & & & & & & 0 & 1 \\
\hline $\mathrm{AC}_{18}$ & & & & & & & & 0 & 1 \\
\hline $\mathrm{AC}_{19}$ & & & & & & & & 0 & 1 \\
\hline $\mathrm{AC}_{20}$ & & & & & & & & 0 & 1 \\
\hline $\mathrm{AC}_{21}$ & & & & & & & & 0 & 1 \\
\hline $\mathrm{AC}_{22}$ & & & & & & & & 0 & 1 \\
\hline $\mathrm{AC}_{23}$ & & & & & & & & 0 & 1 \\
\hline $\mathrm{AC}_{24}$ & & & & & & & & 0 & 1 \\
\hline $\mathrm{AC}_{25}$ & & & & & & & & 0 & 1 \\
\hline $\mathrm{AC}_{26}$ & & & & & & & & 0 & 1 \\
\hline $\mathrm{AC}_{27}$ & & & & & & & & 0 & 1 \\
\hline $\mathrm{AC}_{28}$ & & & & & & & & 0 & 1 \\
\hline RHS & 0 & 0 & 0 & 0 & 0 & 0 & 0 & & \\
\hline Neces. & 1 & 1 & 1 & 1 & 1 & 1 & 1 & & \\
\hline
\end{tabular}

Fonte: Elaborado pelos autores.

ReLAInEP - Revista Latino-America de Inovação e Engenharia de Produção, Curitiba, PR, Brasil, v. 3, n. 4, p. 189-201, 2015. 


\section{ReLAInEP}

A configuração dos parâmetros do Solver do Excel para o problema investigado é apresentada na Figura 5.

FIGURA 5 -Parâmetros do Solver para o problema modelado.

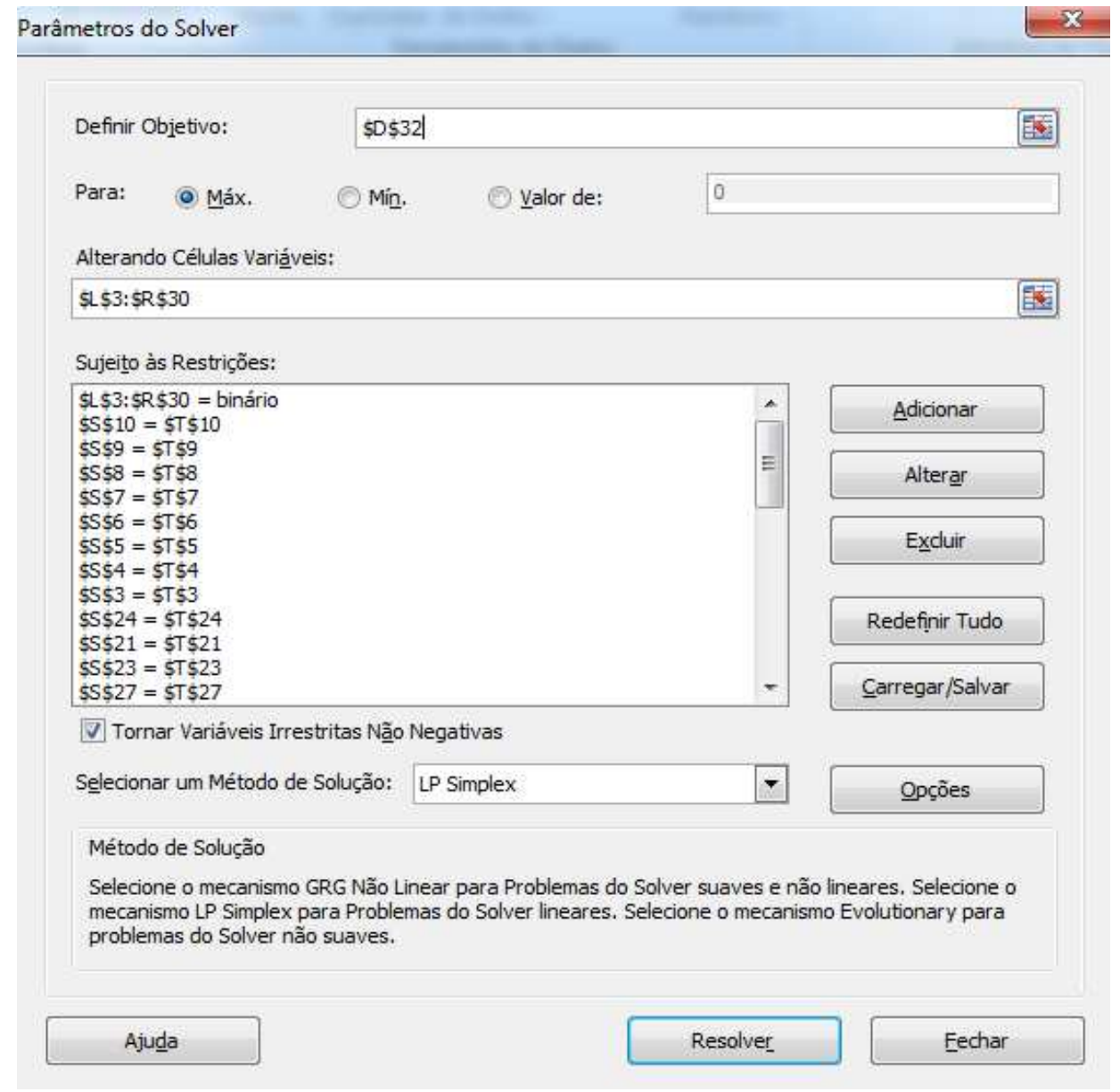

Fonte: Solver do Excel.

A designação de AC fornecida pela execução do modelo, no Solver do Excel, com equipes agrupadas, é apresenta no Quadro 2.

Para obter uma solução com o número de $\mathrm{AC}$ que deverão ser alocados as 12 equipes de apoio, com base nos resultados fornecidos pelo modelo inicial, foram construídas três outros modelos (submodelos). Nos submodelos foram consideradas restrições de disponibilidade, os $\mathrm{AC}$ designados às equipes agrupadas, fornecido pelo modelo inicial. Na solução do modelo referente ao grupo formado pelas equipes de Palestras, Workshop e MesaRedonda, apenas 1 candidato (AC26) foi alocado para as três equipes, portanto, não houve necessidade de criar um submodelo, pois na solução final, o AC26 identificado no modelo inicial fica automaticamente designado para as três equipes.

ReLAInEP - Revista Latino-America de Inovação e Engenharia de Produção, Curitiba, PR, Brasil, v. 3, n. 4, p. 189-201, 2015. 


\section{ReLAInEP}

QUADRO 2 - Número de designações para equipes agrupadas.

\begin{tabular}{|c|c|c|}
\hline Equipe & $\begin{array}{l}\text { Número de } \\
\text { candidatos } \\
\text { designados }\end{array}$ & Acadêmicos Designados \\
\hline Patrocinio/Divulgação & 2 & $\mathrm{AC} 3, \mathrm{AC} 27$ \\
\hline Inscrição/ Certificacãa & 4 & $\mathrm{AC} 2, \mathrm{AC} 11, \mathrm{AC} 17, \mathrm{AC} 24$ \\
\hline $\begin{array}{l}\text { Palestras/ Workshop/ Mesa } \\
\text { redonda }\end{array}$ & 1 & $\mathrm{AC} 26$ \\
\hline Visitas técnicas/ Mini-Cursos & 7 & $\begin{array}{c}\mathrm{AC} 4, \mathrm{AC} 10, \mathrm{AC} 15, \mathrm{AC} 16, \mathrm{AC} 19, \\
\mathrm{AC} 25, \mathrm{AC} 28\end{array}$ \\
\hline Protocolo e Cerimonial & 4 & $\mathrm{AC} 5, \mathrm{AC} 8, \mathrm{AC} 9, \mathrm{AC} 20$ \\
\hline Coffee Break & 6 & $\begin{array}{c}\mathrm{AC} 1, \mathrm{AC} 6, \mathrm{AC} 7, \mathrm{AC} 13, \mathrm{AC} 18, \\
\mathrm{AC} 23\end{array}$ \\
\hline Artigos & 4 & $\begin{array}{l}\mathrm{AC} 12, \quad \mathrm{AC} 14 \\
\mathrm{AC} 21, \mathrm{AC} 22\end{array}$ \\
\hline
\end{tabular}

Fonte: Elaborado pelos autores.

Na construção dos submodelos foram consideras as notas que cada AC atribuiu para as equipes em que foram alocados através da resolução do modelo inicial. Esses novos modelos (submodelos) também foram modelados no Excel e resolvidos pelo Solver.

O primeiro submodelo foi o de Patrocínio e Divulgação. Para essas equipes, de acordo com os resultados do modelo inicial, foram alocados 2 AC. A Figura 6 ilustra os dados do submodelo dispostos no Excel, bem como os resultados fornecidos pelo Solver.

FIGURA 6 - Submodelo: Patrocínio e Divulgação.

\begin{tabular}{|c|c|c|c|c|c|c|c|}
\hline \multicolumn{8}{|c|}{ Patrocínio e Divulgação } \\
\hline & \multicolumn{2}{|c|}{ Dados de Entrada } & & \multicolumn{2}{|c|}{ Alocações } & & \\
\hline AC/EQ & PT & DV & AC/EQ & PT & $\overline{\text { DV }}$ & LHS & Disp. \\
\hline $\mathrm{AC}_{3}$ & 6 & 10 & $\mathrm{AC}_{3}$ & & & 0 & 1 \\
\hline $\mathrm{AC}_{27}$ & 7 & 7 & $\mathrm{AC}_{27}$ & & & 0 & 1 \\
\hline & & & RHS & 0 & 0 & & \\
\hline & $\operatorname{Max} Z=$ & 0 & Neces. & 1 & 1 & & \\
\hline
\end{tabular}

Patrocínio e Divulgação

\begin{tabular}{|c|c|c|c|c|c|c|c|}
\hline & \multicolumn{2}{|c|}{ Dados de Entrada } & & \multicolumn{2}{|c|}{ Alocações } & \multirow[b]{2}{*}{ LHS } & \multirow[b]{2}{*}{ Disp. } \\
\hline AC/EQ & PT & DV & AC/EQ & PT & DV & & \\
\hline $\mathrm{AC}_{3}$ & 6 & 10 & $\mathrm{AC}_{3}$ & 0 & 1 & 1 & 1 \\
\hline $\mathrm{AC}_{27}$ & 7 & 7 & $\mathrm{AC}_{27}$ & 1 & 0 & 1 & 1 \\
\hline & & & RHS & 1 & 1 & & \\
\hline & $\operatorname{Max} Z=$ & 17 & Neces. & 1 & 1 & & \\
\hline
\end{tabular}

Fonte: Elaborado pelos autores.

ReLAInEP - Revista Latino-America de Inovação e Engenharia de Produção, Curitiba, PR, Brasil, v. 3, n. 4, p. 189-201, 2015. 


\section{ReLAInEP}

Com base na resolução deste submodelo, o AC27 foi alocado para a equipe de Patrocínio e o AC3 para a equipe de Divulgação.

O segundo submodelo foi o da equipe de Inscrições e Certificação. Para essas equipes foram alocados 4 AC. A Figura 7 ilustra os dados do submodelo dispostos no Excel, bem como os resultados fornecidos pelo Solver.

Para obter uma solução com o número de AC que deverão ser alocados as 12 equipes de apoio, com base nos resultados fornecidos pelo modelo inicial, foram construídos três outros modelos (submodelos). Nos submodelos foram consideradas restrições de disponibilidade, os $\mathrm{AC}$ designados às equipes agrupadas, fornecido pelo modelo inicial. $\mathrm{Na}$ solução do modelo referente ao grupo formado pelas equipes de Palestras, Workshop e MesaRedonda, apenas 1 candidato (AC26) foi alocado para as três equipes, portanto, não houve necessidade de criar um submodelo, pois na solução final, o AC26 identificado no modelo inicial fica automaticamente designado para as três equipes.

QUADRO 2 - Número de designações para equipes agrupadas.

\begin{tabular}{|ccc|}
\hline Equipe & $\begin{array}{c}\text { Número de } \\
\text { candidatos } \\
\text { designados }\end{array}$ & Acadêmicos Designados \\
\hline $\begin{array}{c}\text { Patrocinio/Divulgação } \\
\text { Inscrição/ Certificação }\end{array}$ & 2 & $\mathrm{AC} 3, \mathrm{AC} 27$ \\
Palestras/ Workshop/ Mesa & 4 & $\mathrm{AC} 2, \mathrm{AC} 11, \mathrm{AC} 17, \mathrm{AC} 24$ \\
redonda & 1 & $\mathrm{AC} 26$ \\
$\begin{array}{c}\text { Visitas técnicas/ Mini-Cursos } \\
\text { Protocolo e Cerimonial }\end{array}$ & 7 & $\mathrm{AC} 4, \mathrm{AC} 10, \mathrm{AC} 15, \mathrm{AC} 16, \mathrm{AC} 19$, \\
Coffee Break & 4 & $\mathrm{AC} 25, \mathrm{AC} 28$ \\
Artigos & 6 & $\mathrm{AC}$, AC8, AC6, AC20 \\
& 4 & $\mathrm{AC} 7, \mathrm{AC} 13, \mathrm{AC} 18$, \\
& & $\mathrm{AC} 12, \mathrm{AC} 14, \mathrm{AC} 21, \mathrm{AC} 22$ \\
\hline
\end{tabular}

Fonte: Elaborado pelos autores.

Na construção dos submodelos foram consideras as notas que cada AC atribuiu para as equipes em que foram alocados através da resolução do modelo inicial. Esses novos modelos (submodelos) também foram modelados no Excel e resolvidos pelo Solver.

O primeiro submodelo foi o de Patrocínio e Divulgação. Para essas equipes, de acordo com os resultados do modelo inicial, foram alocados 2 AC. A Figura 6 ilustra os dados do submodelo dispostos no Excel, bem como os resultados fornecidos pelo Solver.

ReLAInEP - Revista Latino-America de Inovação e Engenharia de Produção, Curitiba, PR, Brasil, v. 3, n. 4, p. 189-201, 2015. 


\section{ReLAInEP}

FIGURA 7 - Submodelo: Inscrição e Certificação.

\begin{tabular}{|c|c|c|c|c|c|c|c|}
\hline \multicolumn{8}{|c|}{ Inscrição e Certificação } \\
\hline \multirow[b]{2}{*}{ AC/EQ } & \multicolumn{2}{|c|}{ Dados de Entrada } & \multirow[b]{2}{*}{ Acadêmico/E } & \multicolumn{2}{|c|}{ Alocações } & \multirow[b]{2}{*}{ LHS } & \multirow[b]{2}{*}{ Disp. } \\
\hline & IN & CT & & IN & CT & & \\
\hline $\mathrm{AC}_{2}$ & 10 & 10 & $\mathrm{AC}_{2}$ & 0 & 1 & 1 & 1 \\
\hline $\mathrm{AC}_{11}$ & 8 & 7 & $\mathrm{AC}_{11}$ & 1 & 0 & 1 & 1 \\
\hline $\mathrm{AC}_{17}$ & 7 & 8 & $\mathrm{AC}_{17}$ & 0 & 1 & 1 & 1 \\
\hline $\mathrm{AC}_{24}$ & 10 & 6 & $\mathrm{AC}_{24}$ & 1 & 0 & 1 & 1 \\
\hline & & & RHS & 2 & 2 & & \\
\hline & $\operatorname{Max} Z=$ & 36 & Neces. & 1 & 1 & & \\
\hline
\end{tabular}

Fonte: Elaborado pelos autores.

Com base na resolução deste submodelo, o AC11 e AC24 foram alocados para a equipe de inscrição e os AC2 e AC17 para a equipe de certificação.

O terceiro submodelo foi o das Visitas Técnicas e Mini-Cursos. Para essas equipes foram alocados 7 candidatos. A Figura 8 ilustra os dados do submodelo dispostos no Excel, bem como os resultados fornecidos pelo Solver.

FIGURA 8 - Submodelo: Visitas Técnicas e Mini-Cursos.

\begin{tabular}{|c|c|c|c|c|c|c|c|}
\hline \multicolumn{8}{|c|}{ Visitas Técnicas e Mini-Cursos } \\
\hline \multirow[b]{2}{*}{ AC/EQ } & \multicolumn{2}{|c|}{ Dados de Entrada } & \multirow[b]{2}{*}{ AC/EQ } & \multicolumn{2}{|c|}{ Alocações } & \multirow[b]{2}{*}{ LHS } & \multirow[b]{2}{*}{ Disp. } \\
\hline & VT & MC & & VT & MC & & \\
\hline $\mathrm{AC}_{4}$ & 10 & 10 & $\mathrm{AC}_{4}$ & & & 0 & 1 \\
\hline $\mathrm{AC}_{10}$ & 9 & 8 & $\mathrm{AC}_{10}$ & & & 0 & 1 \\
\hline $\mathrm{AC}_{15}$ & 10 & 7 & $\mathrm{AC}_{15}$ & & & 0 & 1 \\
\hline $\mathrm{AC}_{16}$ & 9 & 6 & $\mathrm{AC}_{16}$ & & & 0 & 1 \\
\hline $\mathrm{AC}_{19}$ & 8 & 8 & $\mathrm{AC}_{19}$ & & & 0 & 1 \\
\hline $\mathrm{AC}_{25}$ & 10 & 7 & $\mathrm{AC}_{25}$ & & & 0 & 1 \\
\hline \multirow[t]{3}{*}{$\mathrm{AC}_{28}$} & 10 & 10 & $\mathrm{AC}_{28}$ & & & 0 & 1 \\
\hline & & & RHS & 0 & 0 & & \\
\hline & $\operatorname{Max} \mathbf{Z}=$ & 0 & Neces. & 1 & 1 & & \\
\hline
\end{tabular}

ReLAInEP - Revista Latino-America de Inovação e Engenharia de Produção, Curitiba, PR, Brasil, v. 3, n. 4, p. 186-201, 2015. 


\section{ReLAInEP}

Visitas Técnicas e Mini-Cursos

\begin{tabular}{|c|c|c|}
\cline { 2 - 3 } \multicolumn{1}{c|}{} & \multicolumn{2}{c|}{ Dados de Entrada } \\
\hline $\mathbf{A C} / \mathbf{E Q}$ & $\mathbf{V T}$ & $\mathbf{M C}$ \\
\hline $\mathrm{AC}_{4}$ & 10 & 10 \\
\hline $\mathrm{AC}_{10}$ & 9 & 8 \\
\hline $\mathrm{AC}_{15}$ & 10 & 7 \\
\hline $\mathrm{AC}_{16}$ & 9 & 6 \\
\hline $\mathrm{AC}_{19}$ & 8 & 8 \\
\hline $\mathrm{AC}_{25}$ & 10 & 7 \\
\hline $\mathrm{AC}_{28}$ & 10 & 10 \\
\hline \multicolumn{2}{|c|}{$\mathbf{M a x} \mathbf{Z}=$} & 66 \\
\hline
\end{tabular}

\begin{tabular}{|c|c|c|c|c|}
\cline { 2 - 5 } \multicolumn{1}{c|}{} & \multicolumn{2}{c|}{ Alocações } & \multicolumn{1}{c|}{} \\
\hline $\mathbf{A C} / \mathbf{E}$ & VT & MC & LHS & Disp. \\
\hline $\mathrm{AC}_{4}$ & 0 & 1 & 1 & 1 \\
\hline $\mathrm{AC}_{10}$ & 1 & 0 & 1 & 1 \\
\hline $\mathrm{AC}_{15}$ & 1 & 0 & 1 & 1 \\
\hline $\mathrm{AC}_{16}$ & 1 & 0 & 1 & 1 \\
\hline $\mathrm{AC}_{19}$ & 0 & 1 & 1 & 1 \\
\hline $\mathrm{AC}_{25}$ & 1 & 0 & 1 & 1 \\
\hline $\mathrm{AC}_{28}$ & 0 & 1 & 1 & 1 \\
\hline RHS & 4 & 3 & & \\
\hline Neces. & 1 & 1 & & \\
\cline { 1 - 3 } & \multicolumn{2}{|l}{}
\end{tabular}

Fonte: Elaborado pelos autores.

Com base na resolução deste submodelo, os AC10, AC15, AC16 e AC25 foram alocados para a equipe de visitas técnicas e os AC4, AC19 e AC28 para a equipe de MiniCursos.

A partir dos submodelos, foi possível designar os AC para cada equipe conforme mostra o Quadro 3.

QUADRO 3 - Designação dos AC em suas respectivas equipes.

\begin{tabular}{|lcc|}
\hline \multicolumn{1}{|c}{ Equipe } & $\begin{array}{c}\text { Número de candidatos } \\
\text { designados }\end{array}$ & Acadêmicos Designados \\
\hline Artigos (AT) & 4 & AC12, AC14, AC21, AC22 \\
Certificação (CT) & 2 & AC2 e AC17 \\
Coffe Break (CB) & 6 & AC1, AC6, AC7, AC13, AC18, \\
Divulgação (DV) & 1 & AC23 \\
Inscrição (IN) & 2 & AC3 \\
Mesa-Redonda (MR) & 1 & AC11 e AC24 \\
Mini-Cursos (MC) & 3 & AC26 \\
\hline
\end{tabular}

Fonte: Elaborado pelos autores.

\begin{tabular}{|lcc|}
\hline \multicolumn{1}{|c}{ Equipe } & $\begin{array}{c}\text { Número de candidatos } \\
\text { designados }\end{array}$ & Acadêmicos Designados \\
\hline Palestras (PL) & 1 & AC26 \\
Patrocínio (PT) & 1 & AC27 \\
Protocolo e Cerimonial (PC) & 4 & AC5, AC8, AC9, AC20 \\
Visitas Técnicas (VT) & 4 & AC10, AC15, AC16 e AC25 \\
Workshop (WS) & 1 & AC26 \\
\hline
\end{tabular}

Fonte: Elaborado pelos autores.

ReLAInEP - Revista Latino-America de Inovação e Engenharia de Produção, Curitiba, PR, Brasil, v. 3, n. 4, p. 179-201, 2015. 


\section{ReLAInEP}

\section{CONSIDERAÇÕES FINAIS}

Uma das atividades geridas por comitês organizadores de eventos científicos é a designação de todas as tarefas às pessoas que as possam realizar.

Para realizar a designação de tarefas aos Acadêmicos do Curso de Engenharia de Produção Agroindustrial (EPA) da Unespar/Campus de Campo Mourão, que se candidataram a compor a equipe organizadora, propôs-se neste trabalho a aplicação da Programação Linear fundamentada no Problema de Atribuição de Tarefas, buscando encontrar a distribuição ótima dos candidatos entre as equipes de apoio a Comissão Organizadora do VIII EEPA e II ENEPRO.

O problema em estudo, por ser tratar de um problema de pequeno porte, pode ser resolvido no Solver do Excel. O modelo matemático proposto mostrou ser eficiente, obtendo uma solução satisfatória tanto nas capacidades dos candidatos, quanto na demanda necessária ao evento.

Vale ressaltar que em eventos que apresentam comissões organizadoras com maiores quantidades de candidatos em relação ao apresentado nesse artigo, talvez sejam necessárias outras ferramentas para a otimização do problema de alocação de tarefas, pois o Solver resolve problemas com até 200 variáveis. Sugere-se para trabalhos futuros, que estudos similares a este, sejam realizados em Softwares especializados, como por exemplo, o Software LINDO.

\section{REFERENCIAS}

ARENALES, Marcos Nereu.; ARMENTANO, Vinícius.; MORABITO, Reinaldo; YANASSE, Horácio. Pesquisa Operacional. Rio de Janeiro: Elsevier, 2007.

BARBOSA, Gabriela Campos Costa. Uma Abordagem Híbrida para Alocação de Profissionais em Projeto de Tecnologia da Informação. 2009. 72 f. Tese (Mestrado em Informática Aplicada) - Universidade de Fortaleza (UNIFOR), Fortaleza, 2009.

COSTA, Ana Paula Cabral Seixas. SILVA, Lúcio Camara . BASTOS, Raquel Neves. Modelo de decisão para alocação de Recursos Humanos em projetos de Sistemas de Informação. In: XXIX ENEGEP, 2009, Salvador. Anais XXIX ENEGEP, 2009.

GOLDBARG, Marco Cesar; LUNA, Henrique Pacca Loureiro. Otimização combinatória e programação linear. $2^{\mathrm{a}}$ Ed. Rio de Janeiro: Campus, 2005.

LACERDA, Aureliana Lopes. WEBER, Claudiane. PORTO, Marchelly Pereira. SILVA, Romário Antunes da. A importância dos eventos científicos na formação acadêmica: estudantes de Biblioteconomia. Revista ABC, Florianópolis, v. 13, n. 1, p. 130-144, jan./jun, 2008.

LANCHTERMACHER, G. Pesquisa Operacional na Tomada de Decisões: modelagem em Excel. $3^{\text {a }}$ edição. Rio de Janeiro: Campus, 2007.

MARQUES, Anna Beatriz. RODRIGUES, Rosiane. PRIKLADNICKI, Rafael. CONTE, Taynara. Alocação de Tarefas em Projetos de Desenvolvimento Distribuído de Software: Análise das Soluções Existentes. In: II Congresso Brasileiro de Software: Teoria e Prática, V

ReLAInEP - Revista Latino-America de Inovação e Engenharia de Produção, Curitiba, PR, Brasil, v. 3, n. 4, p. 179-201, 2015. 


\section{ReLAInEP}

Workshop de Desenvolvimento Distribuído de Software, 2011, São Paulo. CBSoft II Congresso Brasileiro de Software: Teoria e Prática, 2011. v. 11. p. 1-8.

MELLO, João Carlos Correia Baptista Soares. GOMES, Eliane Gonçalves. LETA, Fabiana Rodrigues. MELLO, Maria Helena Campos Soares. Algoritmo de alocação de recursos discretos com análise de envoltória de dados. Revista Pesquisa Operacional, Rio de Janeiro, v. 26, n. 2, mai./ago. 2006.

MORAIS, Márcia de Fátima. Introdução à Pesquisa Operacional: Apostila. Disciplina de Introdução à Pesquisa Operacional, Curso de Engenharia de Produção Agroindustrial, Departamento de Engenharia de Produção, Campus de Campo Mourão, Universidade Estadual do Paraná (UNESPAR), Campo Mourão/PR, 2013.

RODRIGUES, Laís Bássame. VIEIRA, Fabiano Bahia Pereira.; AGUSTINI, Edson. O método húngaro de otimização para o problema da alocação de tarefas. Universidade Federal de Uberlândia, FAMAT em revista, número 4, abril de 2005.

SILVA, Philipe Jorge. DIAS, Igor Norberto Bedeti. ALVARENGA, Larissa Assis. CAMPOS, Magno Silvério. SILVA, André Luís. Alocação de sessões de artigos em eventos acadêmicos: modelo e estudo de caso. Revista Eletrônica Pesquisa Operacional para o Desenvolvimento. v.6, n.1, p. 54-66, Jan. a Abr. de 2014.

SUCENA, Marcelo Prado. Programação Linear Inteira: Apostila. Disciplina de Pesquisa Operacional II, Curso de Engenharia de Produção, Departamento de Engenharia de Produção, Universidade Estácio de Sá (UES), Rio de Janeiro, 2012.

TAHA, H. M. Pesquisa Operacional. 8a. edição. São Paulo: Pearson Prentice Hall, 2008.

TEIXEIRA, Vinícius Garcia. Aplicação de programação linear na alocação de vagões gôndola para o transporte de ferro gusa na mrs logística s.a. 2011. 56 f. Tese (Graduação em Engenharia de Produção) - Universidade Federal de Juiz de Fora. Juiz de Fora, 2011.

ReLAInEP - Revista Latino-America de Inovação e Engenharia de Produção, Curitiba, PR, Brasil, v. 3, n. 4, p. 179-201, 2015. 
ReLAInEP - Revista Latino-America de Inovação e Engenharia de Produção, Curitiba, PR, Brasil, v. 3, n. 4, p. 179-201, 2015. 